\title{
Stand und Perspektive der Kurzzeitpflege
}

Janina Kutzner und Miriam Räker

Inhaltsverzeichnis

9.1 Hintergrund -132

9.2 Funktion und Bedeutung der Kurzzeitpflege - 133

9.3 Angebot und Inanspruchnahme der Kurzzeitpflege - 135

9.4 Handlungsfelder zur Stärkung der Kurzzeitpflege - 137

$9.5 \quad$ Fazit -142

Literatur - 143 


\section{- Zusammenfassung}

Die Kurzzeitpflege (KZP) und ihre Funktion standen bislang nur unzureichend im Fokus wissenschaftlicher Untersuchungen. Der Beitrag betrachtet die gesetzlich zugeschriebene Bedeutung der KZP und hinterfragt, inwieweit diese Versorgungsform einer sowohl präventiven als auch rehabilitativen Funktion gerecht wird respektive gerecht werden kann. Dazu wird vor dem Hintergrund des bestehenden sozialrechtlichen Rahmens der Fokus auf die quantitative Verfügbarkeit von KZP-Plätzen sowie auf die qualitative und inhaltliche Ausrichtung von Angeboten der KZP gerichtet. Wenngleich es an wissenschaftlicher Evidenz fehlt, lässt die Betrachtung der verschiedenen Organisationsformen der KZP vermuten, dass solitäre KZP-Einrichtungen der wichtigen Weichenstellerfunktion der KZP sowie einer präventiven und rehabilitativen Funktion am ehesten gerecht werden können. Demgegenüber stehen jedoch vielfach betriebswirtschaftliche und strukturelle Herausforderungen. Besonders mit Blick auf einen geriatrisch rehabilitativen Ansatz im Rahmen der KZP sind sozialrechtliche Hürden und in der Folge Versorgungsdefizite anzunehmen. Die Notwendigkeit für weitergehende Reformbemühungen wird bei der Darstellung von Möglichkeiten und Potenzialen zur Stärkung und Weiterentwicklung der KZP deutlich.

Short-term care (STC) and its function have so far been insufficiently the focus of scientific research. The article analyses the significance attributed to short-term care by law and questions the extent to which this form of care fulfils or can fulfill a preventive as well as a rehabilitative function. Against the background of the existing social legal framework, the authors focus the quantitative availability of STC places as well as the qualitative and content-related orientation of STC. Although there is a lack of scientific evidence, an examination of the various organisational forms of STC suggests that solitary STC facilities are best able to fulfil the important function of setting the course and at the same time provide pre- vention and rehabilitation. However, they are often faced with economic and structural challenges. Particularly with regard to a geriatric rehabilitative approach within the framework of STC, there might be some socio-legal obstacles and resulting deficits. The presentation of possibilities and potentials for strengthening and further developing STC reveals that more far-reaching reform efforts are called for.

\subsection{Hintergrund}

Die Anforderungen an die Kurzzeitpflege (KZP) nach § 42 Sozialgesetzbuch (SGB) XI sind groß. Grundsätzliches Ziel der KZP ist es, den Verbleib in der Häuslichkeit im Anschluss an eine Krankenhausbehandlung sowie bei Krisensituationen des häuslichen Versorgungsettings weiter zu ermöglichen. Der Kurzzeitpflege wird insofern eine präventive und letztlich auch rehabilitative Funktion zugedacht. Die Möglichkeit der vorübergehenden Pflege und Betreuung einer pflegebedürftigen Person in einer Einrichtung der KZP kann dazu beitragen, pflegende Angehörige zu entlasten und häusliche Pflegearrangements zu stabilisieren. Stationäre Langzeitpflege kann und soll durch den Einsatz von KZP hinausgezögert oder sogar verhindert werden (Deutscher Bundestag 2019; Deckenbach et al. 2013). Das liegt sowohl im Interesse der Pflegebedürftigen im Sinne ihres Wunsches nach Verbleib in der eigenen Häuslichkeit (Rothgang et al. 2012) als auch im Interesse der Beitragszahler, da Kosten vermieden bzw. reduziert werden können. Die KZP orientiert sich in der Folge an dem Prinzip der Sozialen Pflegeversicherung (SPV) ,ambulant vor stationär" (§ 3 SGB XI; § 13 SGB XI). Trotz unterschiedlicher Reformbemühungen in den vergangenen Jahren und einer dadurch geschaffenen Aufmerksamkeit für die Thematik scheint die KZP ihrer Funktion und auch ihren Möglichkeiten nicht ausreichend gerecht $\mathrm{zu}$ werden, was sich u. a. in zu niedrigen Rückführungsquoten von Patientinnen und Patienten nach 
einem Krankenhausaufenthalt in die eigene Häuslichkeit widerspiegelt (u. a. Becker et al. 2020). Dieser Umstand ist vor dem Hintergrund des enormen Potenzials, den die KZP bietet, sowie eines möglichen Wandels der Klientel mit komplexen Versorgungsbedarfen besonders bedeutsam. Der Beitrag wendet sich vor diesem Hintergrund der Frage zu, inwiefern das bestehende Leistungs- und Sozialrecht dem Anspruch einer präventiven und rehabilitativen Versorgung in der KZP entgegensteht. Ebenso wird der Frage nach einer ausreichenden quantitativen Verfügbarkeit von - vorrangig - solitären KZP-Plätzen nachgegangen. Hierzu wird im ersten Schritt die derzeitige sozialrechtliche Ausgestaltung aufgezeigt, anschließend werden Problemfelder sowie Handlungsoptionen zur Stärkung und Weiterentwicklung der KZP diskutiert. Ziel des Beitrags ist es, Problembereiche ebenso wie Möglichkeiten und Potenziale der KZP aufzuzeigen und somit die inhaltliche Diskussion um Aspekte der strukturellen Ausgestaltung zu erweitern.

\subsection{Funktion und Bedeutung der Kurzzeitpflege}

Bereits seit den Siebzigerjahren existiert die KZP als reine Urlaubspflege. Erstmals sozialrechtlich verankert wurde das Versorgungsangebot 1989 mit Einführung der Schwerpflegebedürftigkeit nach $\S 56$ SGB V, Kostenträger waren die gesetzlichen Krankenkassen. Mit der Einführung der SPV 1995 wurde die Kurzzeitpflege in $\S 42$ SGB XI geregelt. Hiervon abzugrenzen ist die Verhinderungspflege nach $\S 39$ SGB XI. Seit Inkrafttreten des Pflegestärkungsgesetzes (PSG) I zum 1. Januar 2015 können diese beiden Leistungsarten gegeneinander angerechnet werden: $50 \%$ der Mittel aus der KZP können in die Verhinderungspflege und $100 \%$ des Anspruchs auf Verhinderungspflege in die KZP ( $\$ 42$ SGB XI) übertragen werden. Zudem haben seit 2016 mit Inkrafttreten des Krankenhausstrukturgesetzes (KHSG) auch Personen ohne Pflegebedürftigkeit im Sinne des SGB XI sowie Pflegebedürftige des Pflegegrades 1 nach § 39c SGB V einen Anspruch auf KZP.

Anspruch auf KZP besteht, wenn aufgrund von akuten Krankheitsereignissen vorübergehend Hilfe in einem Ausmaß benötigt wird, dass häusliche Krankenpflege nicht ausreicht. Zumeist ist dies nach einem Krankenhausaufenthalt, nach einer ambulanten Operation oder nach einer ambulanten Krankenhausbehandlung der Fall. Der KZP wird in dieser Funktion folglich eine wichtige ,Weichenstellerfunktion“" zuteil (siehe • Abb. 9.1), da sie an der Schnittstelle individueller Pflegeverläufe zwischen Akutaufenthalt oder akuter Versorgungssituation und dem weiteren Versorgungsweg angesiedelt ist (Becker et al. 2020). Gleichzeitig hat die KZP ähnlich wie die Verhinderungspflege nach $\S 39$ SGB XI die Funktion, kurzzeitigen stationären Ersatz der häuslichen Pflege bei Verhinderung oder zur Entlastung der pflegenden Angehörigen zu sichern. Die Verhinderungspflege unterscheidet sich von der KZP insofern, als dass sie ausschließlich zu Hause erbracht wird und bei Bedarf nur stundenweise in Anspruch genommen werden kann.

Während die Verhinderungspflege in der Regel. planbar und ausschließlich eine Ersatzpflege ist, geht die KZP in ihrer Funktion insbesondere im Fall von Krankenhausübergangspflege deutlich darüber hinaus. Innerhalb einer relativ kurzen Zeitspanne wird eine hohe pflegerische Versorgungsqualität und zugleich eine Klärung der weiteren Versorgung im Sinne eines systematischen Überleitungsmanagements (ÜLM) gefordert (Deckenbach und Pflug 2019). Ferner ist bekannt, dass bei der Klientel, die in der KZP versorgt wird, ein hoher Bedarf, aber auch ein hohes Potenzial für Rehabilitation und therapeutische Aktivierung vorliegt (u.a. Janßen 2018; Rothgang et al. 2014). Zu dem Umfang, in dem potenziell gebotene Rehabilitationsmaßnahmen unterbleiben, liegen jedoch keine ausreichenden Studien vor (Rothgang et al. 2014; Lübke 2015 zit. n. Ehmen et al. 2019). Der Anspruch an 


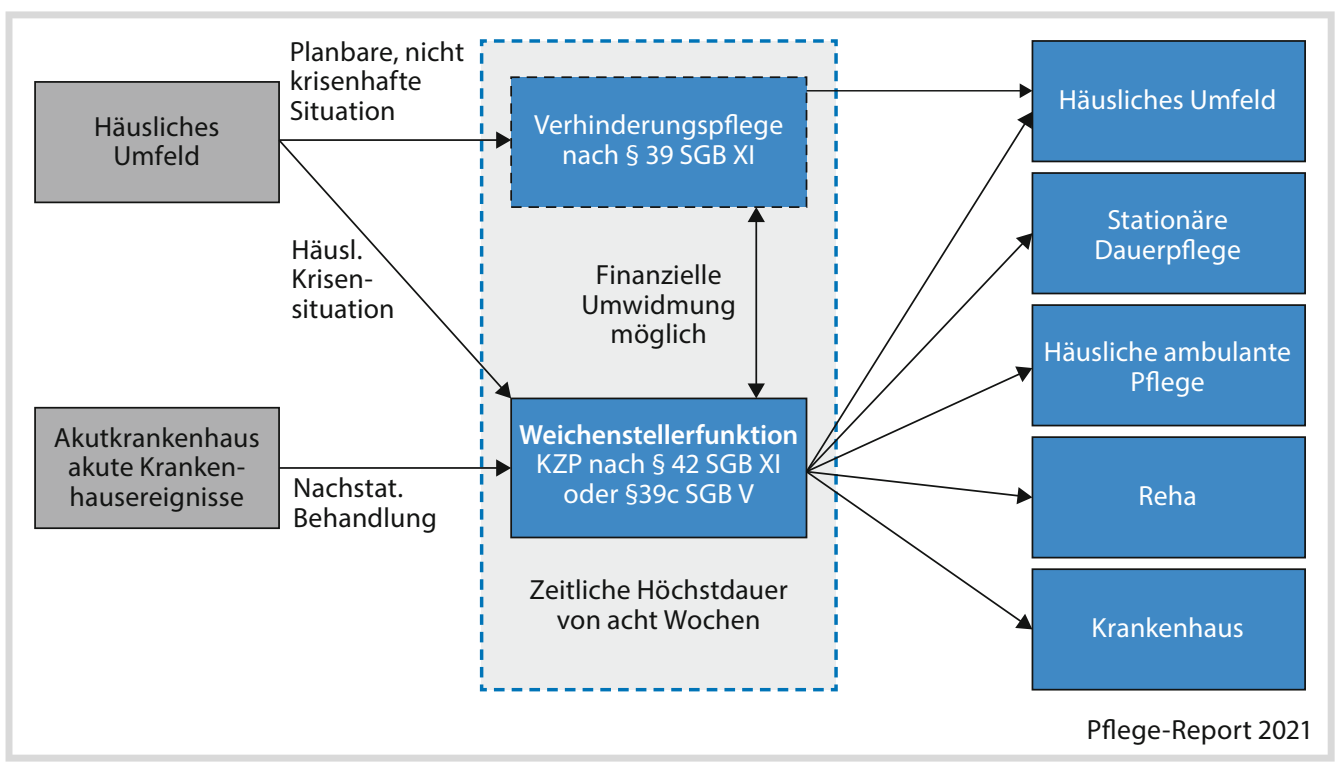

- Abb. 9.1 Versorgungspfade und Weichenstellerfunktion der Kurzzeitpflege. (Quelle: eigene Darstellung in Anlehnung an Braeseke et al. 2017)

KZP geht in der Folge vielfach über die Grundund Behandlungspflege nach SGB XI hinaus und macht damit die Abgrenzung zur Verhinderungspflege deutlich. Gerade die Übergangsphase nach akutstationärer Behandlung ist durch einen erhöhten Bedarf an aktivierender Pflege und rehabilitativen Therapien, insbesondere Physio-Ergotherapie, gekennzeichnet (Deckenbach et al. 2013). Allerdings wird im Rahmen der KZP aber nur eine pflegerische Versorgung finanziert, also keine Rehabilitationsmaßnahmen (Becker et al. 2020). Die Pflegekasse übernimmt die pflegebedingten Aufwendungen einschließlich der Aufwendungen für Betreuung sowie für Leistungen der medizinischen Behandlungspflege bis $\mathrm{zu}$ einem Gesamtbetrag von $1.612 €$ im Kalenderjahr. Dies entspricht dem Betrag, der auch bei der Verhinderungspflege gewährt wird. Entsprechend spiegeln sich der explizit präventive und rehabilitative Anspruch und die daraus für die pflegerische Versorgung resultierenden Anforderungen sowie die spezifischen Bedarfe der KZP-Klientel insbesondere mit Blick auf Personalqualifikation und -ausstattung im aktuellen Regulierungsrahmen, auch in den Landes- rahmenverträgen (bspw. Rahmenvertrag KZP Baden-Württemberg), nicht angemessen wider. Pflegeleistungen gehen nicht über die aktivierende Pflege nach $\S 43 \mathrm{~b}$ SGB XI hinaus, Zusatzleistungen sind im Rahmen von $\S 88$ SGB XI möglich, müssen von den Pflegebedürftigen aber selbst getragen werden.

Bei der Versorgung von Pflegebedürftigen in der postakuten Phase, also an den Übergängen zu anderen Sektoren und/oder Versorgungsbereichen, sind multiprofessionelle Ansätze von Bedeutung, doch gerade hier existieren hohe (vergütungsrechtliche) Barrieren (Deckenbach et al. 2013; Deckenbach und Pflug 2019). Die Trennung der Leistungsbereiche nach SGB V und SGB XI sowie die damit einhergehende Finanzierungssystematik respektive -zuständigkeit (Behandlungs- und Grundpflege) verschärfen das Problem. In der Gesamtschau ist folglich das Fehlen eines allgemeinen und geriatrischen rehabilitativen Ansatzes in der sozialrechtlichen Verankerung der KZP zu konstatieren.

Dies steht im Kontrast zu Studien, die auf einen hohen Bedarf an Leistungen der medizinischen Rehabilitation bei Personen in KZP 
hinweisen (u. a. Janßen 2018; Rothgang et al. 2014). 2018 wurde erstmals eine empirische Analyse des Bedarfs an Leistungen der medizinischen Rehabilitation bei Bewohnenden und Kurzzeitpflegegästen in Einrichtungen der stationären Pflege durchgeführt (Janßen 2018). Bei 22,7\% der Langzeit-Bewohnenden und bei 32,6\% der Kurzzeitpflegegäste konnte ein solcher Bedarf festgestellt werden (ebd.). Bei ca. $30 \%$ der geriatrischen Patientinnen und Patienten, die aus Akutkliniken in die KZP entlassen werden, lagen zum Zeitpunkt der Aufnahme Rehabilitationsbedarfe mit Besserungspotenzial vor, trotz nicht gegebener Rehabilitationsfähigkeit im Sinne einer Indikation zur stationären Rehabilitation nach $\S 40$ SGB V (ebd.).

Nach einer Untersuchung von Rothgang et al. erfolgt mit fast $60 \%$ der Großteil der KZP-Aufnahmen im Anschluss an einen Krankenhausaufenthalt. Im weiteren Versorgungsverlauf werden $42 \%$ dieser Patientinnen und Patienten in die vollstationäre Dauerpflege übergeleitet (Rothgang et al. 2015). Mit Blick auf die geringe Rückführung von Patientinnen und Patienten nach einem Krankenhausaufenthalt in die Häuslichkeit von weniger als $40 \%$ (ebd.) stellt sich zunehmend die Frage, inwiefern das Rehabilitationspotenzial der einzelnen Teilgruppen, die KZP in Anspruch nehmen, ausreichend aktiviert wird und eine bedarfsgerechte Versorgung vorliegt. Der Grundsatz „Reha vor Pflege“" findet in der Folge im Kontext der KZP bislang nur unzureichend Anwendung. $\mathrm{Zu}$ vermuten ist, dass nur bei einer Minderheit der Betroffenen funktionelle Einschränkungen stabilisiert oder verbessert werden und das häusliche Settings nicht ausreichend auf die Versorgung der pflegebedürftigen Personen gestärkt und vorbereitet wird, damit eine Rückkehr ins häusliche Umfeld möglich ist. Die eigentliche Funktion der KZP wird dadurch nicht erfüllt (Becker et al. 2020).

\subsection{Angebot und Inanspruchnahme der Kurzzeitpflege}

Das derzeitige Angebot an KZP kann in zwei grundlegende Organisationsformen - solitäre und eingestreute KZP - unterteilt werden. Aufgrund der jeweiligen Landespflegegesetze der Bundesländer, welche die Angebotsstruktur von KZP-Einrichtungen regeln, unterscheiden sich die Angebotsformen in den einzelnen Ländern zum Teil erheblich. In NordrheinWestfalen können beispielsweise in vollstationären Pflegeeinrichtungen Betten für KZP als sogenannte „eingestreute Kurzzeitpflege“ oder als eigene Abteilung innerhalb des Hauses - separate Kurzzeitpflegeplätze - freigehalten werden. Eingestreute KZP meint Plätze in einer vollstationären Dauerpflegeeinrichtung, die kurzfristig, flexibel für KZP genutzt werden können. In der Mehrzahl der Bundesländer ist das Angebot eingestreuter KZP möglich. Dagegen werden KZP-Plätze in Berlin und Sachsen fast ausschließlich in sogenannten „,solitären Kurzzeitpflegeeinrichtungen“ angeboten. In Hamburg und Schleswig-Holstein gibt es wiederum ausschließlich eingestreute bzw. separate KZP-Plätze in vollstationären Pflegeeinrichtungen (Braeseke et al. 2017). Trotz der bestehenden Unterschiede in einzelnen Bundesländern werden KZP-Plätze bundesweit überwiegend als flexibel nutzbare, eingestreute KZP in stationären Pflegeeinrichtungen angeboten.

Die Verantwortung für die Pflegebedarfsplanung liegt im Aufgabenbereich der Länder ( 9 SGB XI), welche die Verantwortung oftmals mittels entsprechender Landespflegegesetze an die Kommunen weitergeben. Eine flächendeckende Pflegebedarfsplanung liegt in den einzelnen Ländern bislang nicht vor, auch fehlt es an einheitlichen Kriterien (Hackmann 
et al. 2014). ${ }^{1}$ Eine unzureichende Pflegebedarfsplanung der Länder und Kommunen führt dazu, dass die vor Ort benötigten Kapazitäten nur schwer abzuschätzen sind, dies gilt auch für die KZP (Vonau o.J.; Braeseke et al. 2017). Für die Bedarfsplanung im Bereich der KZP ergibt sich zudem eine besondere Schwierigkeit, da sich eine Bestandsaufnahme von KZP-Plätzen aufgrund der verschiedenen Organisationsformen nach solitärer, separater und eingestreuter KZP schwierig gestaltet (Braeseke et al. 2017) und eine Festlegung von Bedarfsrichtwerten erschwert. Überdies werden in (amtlichen) Erhebungen, wie bspw. der Pflegestatistik, nur die KZP-Plätze in solitären oder separaten KZP-Einrichtungen erfasst (ebd.). Dies kann als ein Grund dafür angesehen werden, dass viele Bundesländer keine verbindlichen Richtwerte für KZP-Plätze aufweisen (Hartmann 2002). Für die KZP lassen sich daher nur schwer verlässliche Aussagen zum tatsächlich vorhandenen Angebot an KZP-Plätzen treffen. Ungeachtet der schwachen Datenbasis zeigt die Pflegestatistik, dass sich die Anzahl der solitären und separaten KZP-Einrichtungen in den letzten zehn Jahren fast halbiert hat (von 279 auf 148 KZP-Einrichtungen) (Statistisches Bundesamt 2021). Hingegen hat die Inanspruchnahme an allen KZP-Plätzen insgesamt im selben Zeitraum um gut $72 \%$ zugenommen. Die Studie des IGES-Instituts zur Situation der KZP in NRW kam zu dem Schluss, dass in nur 15 von insgesamt 54 Kreisen und kreisfreien Städten ein ausreichendes Angebot an KZP-Plätzen zur Verfügung stand. Der vorhandene Bedarf an KZP-Plätzen hätte der Studie zufolge nur durch einen Mix aus solitären bzw. separaten und eingestreuten KZP-Plätzen gedeckt werden können (Braeseke et al. 2017).

Die Ursachen für regional defizitäre KZPAngebote sind vielfältig. Neben unattraktiven

1 In NRW und Rheinland-Pfalz wurde bspw. die Zuständigkeit für die Pflegebedarfsplanung an die Kommunen übertragen (Hackmann et al. 2014). Dort sind in den jeweiligen Landespflegegesetzen detaillierte und verpflichtende Vorgaben zur Ausgestaltung der kommunalen Pflegestrukturplanungen festgehalten.
Finanzierungsmodellen und dem Fachkräftemangel in der Pflege stellt sich der Betrieb von solitären KZP-Einrichtungen nach der Untersuchung Braeseke et al. (2017) als wirtschaftlich schwierig dar (Deckenbach und Pflug 2019; siehe auch Brettschneider 2019). Die Differenzierung zwischen solitärer, separater und eingestreuter KZP ist vergütungsrechtlich und betriebswirtschaftlich relevant. Die Aufwendungen für solitäre KZP-Einrichtungen werden höher vergütet als für eingestreute oder separate KZP-Plätze, die Investitionskosten bei solitären KZP-Einrichtungen fallen deutlich geringer aus (Braeseke et al. 2017). Bei eingestreuten KZP-Plätzen können drohende Verluste durch z. B. stark schwankende Auslastungszahlen durch flexible Nutzung reduziert werden. Nicht genutzte Plätze solitärer KZP-Einrichtungen können nicht anderweitig genutzt werden und sind demnach kostspielig, auch können bestehende Fixkosten nicht reduziert werden. Flexibel eingestreute KZP-Plätze in vollstationären Pflegeeinrichtungen bergen wiederum das Risiko, dass diese im Bedarfsfall bereits mit vollstationären Bewohnenden belegt sind (ebd.). Eingestreute KZP-Plätze sind für stationäre Pflegeeinrichtungen ebenfalls wenig wirtschaftlich, können jedoch als „strategisches Akquiseinstrument“ genutzt werden, wenngleich die gleichzeitig hohe Nachfrage nach Dauerpflege dies unattraktiv macht (Heinzen et al. 2020).

Zwischen 2015 und 2019 betrug das Wachstum an Leistungsempfängern und -empfängerinnen von KZP (• Tab. 9.1) 9\%. Demgegenüber hat sich die Anzahl an Leistungsempfängern und -empfängerinnen der SPV im ambulanten Sektor im gleichen Zeitraum um $29 \%$ erhöht. Die Entwicklung der Anzahl an pflegebedürftigen Leistungsempfänger und -empfängerinnen spiegelt sich in der Folge nicht in der Anzahl der Inanspruchnahme an KZP wider. Die Entwicklung der Inanspruchnahme der KZP bleibt demnach hinter den Erwartungen zurück, trotz unterschiedlicher reformpolitischer Maßnahmen wie dem PSG I und dem Versorgungsstrukturgesetz. Verglichen mit den vorangegangenen vier Jah- 
- Tab. 9.1 Entwicklung der Inanspruchnahme von KZP 2010 bis 2019. (Datenquelle: Amtliche Statistik PG 1 und PG 22010 bis 2021 (BMG 2021))

\begin{tabular}{|l|l|l|}
\hline Jahr & $\begin{array}{l}\text { Leistungsempfänger KZP } \\
\text { im Jahresdurchschnitt }\end{array}$ & $\begin{array}{l}\text { Leistungsempfänger ambulant insgesamt } \\
\text { am 31.12. des Jahres }\end{array}$ \\
\hline 2010 & 17.255 & 1.577 .844 \\
\hline 2011 & 17.692 & 1.602 .070 \\
\hline 2012 & 18.427 & 1.667 .108 \\
\hline 2013 & 19.749 & 1.739 .337 \\
\hline 2014 & 20.595 & 1.818 .052 \\
\hline 2015 & 23.853 & 1.907 .095 \\
\hline 2016 & 24.632 & 1.974 .197 \\
\hline 2017 & 25.974 & 2.359 .035 \\
\hline 2018 & 26.639 & 2.561 .991 \\
\hline 2019 & 26.299 & 2.698 .092 \\
\hline $\begin{array}{l}\text { a Ab 2017 ohne Pflegegrad 1 } \\
\text { Pflege-Report 2021 }\end{array}$ & & \\
\hline
\end{tabular}

ren 2011 bis 2015 betrug die Wachstumsrate der Inanspruchnahme an KZP zumindest $26 \%$, während gleichzeitig die Zahl der Leistungsempfänger und -empfängerinnen insgesamt um $16 \%$ und damit deutlich geringer anstieg.

\subsection{Handlungsfelder zur Stärkung der Kurzzeitpflege}

KZP muss entsprechend $\S 42$ SGB XI einen hochkomplexen Versorgungsauftrag mit fachlich und organisatorisch anspruchsvollen Aufgaben gerecht werden, die sich nicht zuletzt u. a. in der Weichenstellerfunktion widerspiegeln (Deckenbach und Pflug 2019). Um dem sozialrechtlich verankerten Versorgungsauftrag gerecht werden zu können, muss KZP - abgesehen davon, dass eine Ausweitung des quantitativen Angebots an KZP-Plätzen notwendig ist - inhaltlich neu gedacht und gestärkt werden (Vonau o.J.).

\section{- Quantitative Ausweitung}

Zur Stärkung der KZP bedarf es grundsätzlich einer Ausweitung des quantitativen Angebots an KZP-Plätzen. Erfahrungen aus der Praxis und aus versorgungswissenschaftlicher Perspektive weisen darauf hin, dass feste solitäre KZP-Plätze im Gegensatz zu flexiblen eingestreuten Plätzen für eine nachhaltige, qualitätsorientierte Versorgung von Bedeutung sind. Bei eingestreuten KZP-Plätzen in stationären Einrichtungen besteht die Gefahr, dass diese im Zuge steigender Nachfrage nach vollstationärer Dauerpflege immer weniger zur Verfügung stehen (Deckenbach und Pflug 2019). Für stationäre Pflegeeinrichtungen ist es hingegen aufgrund der derzeitigen Refinanzierungsbedingungen oftmals wenig lukrativ, ein entsprechendes Angebot an „,fixen“ Kurzzeitpflegeplätzen vorzuhalten (Brettschneider 2019). Es besteht die Gefahr, dass eingestreute Betten im Pflegealltag untergehen, mit der Folge, dass der oftmals komplexe und heterogene Versorgungsbedarf unzureichend beachtet wird (Deckenbach und Pflug 2019). 


\section{- - Finanzierung und Vergütung}

Um das quantitative Angebot an KZP-Plätzen, insbesondere an solitären Plätzen, nachhaltig auszubauen, sind vorrangig finanzielle Anreize und eine wirtschaftlich tragfähige Finanzierung der Einrichtungen notwendig (Braeseke et al. 2017; Deckenbach und Pflug 2019). Dies beinhaltet u. a. veränderte Refinanzierungsregelungen, um Verluste durch Auslastungsschwankungen zu reduzieren (Braeseke et al. 2017). Auch eine zielgerichtete Förderung, etwa in Form der Investitionskostenförderung durch die Länder - dies ist bislang erst in acht Bundesländern der Fall -, muss geschaffen werden. Ebenso sind höhere Pflegesätze für die oftmals sehr aufwendige Kurzzeitpflege notwendig (Braeseke et al. 2017; Deckenbach und Pflug 2019). Hieran anknüpfend müssen andere Vergütungsmöglichkeiten geschaffen werden, z. B. in Form von höheren Leistungspauschalen, die grundsätzlich den Einbezug interdisziplinärer und vor allem integrierter Versorgung berücksichtigen, aber auch den Organisationsaufwand, der mit der KZP verbunden ist (ebd.).

\section{- Pflegerische Bedarfsplanung und die Rolle der Kommunen}

Grundlage eines zielgereichten KZP-Angebots, sowohl qualitativ als auch quantitativ, ist eine funktionierende Pflegebedarfsplanung, verbunden mit einer inhaltlichen Anpassung und Erweiterung des Versorgungsauftrages der KZP im Sozialrecht. Durch die in der aktuellen Form bestehende Pflegestrukturplanung der Länder und Kommunen sind die vor Ort benötigten Kapazitäten nur schwer kalkulierbar, nicht nur im Hinblick auf quantitative Aspekte, wie die reine Betten- respektive Platzzahl, sondern auch mit Blick auf die Frage nach bedarfsgerechten Versorgungsansätzen (SVR 2014). Daneben stellt sich die Frage nach der Erweiterung der Handlungsmöglichkeiten der Akteure vor Ort, die die Planung, Steuerung und Koordinierung der pflegerischen Versorgungsstrukturen verantworten. Die Stärkung der Rolle der Kommunen, wie sie in der pflegerischen Versorgung seit vielen Jahren gefordert wird (Blom und Görres 2012; Naegele 2010; CDU/CSU/SPD 2013), könnte auch für die Weiterentwicklung der KZP von zentraler Bedeutung sein. Durch eine Stärkung kommunaler Verantwortlichkeit und Handlungsfähigkeit könnte passgenauer auf regionalspezifische Bedarfe reagiert und die Versorgungsgestaltung vor Ort entsprechend angepasst werden. Damit der bereits heute vorhandene Flickenteppich in der KZP dadurch nicht weiter verstärkt wird, braucht es klare Rahmenbedingungen für die Ausgestaltung der KZP. Ungeachtet der Problematik im Bereich der Pflegebedarfsplanung verfügen die Kommunen derzeit kaum über Regulierungs- und Steuerungsmöglichkeiten mit Blick auf das Angebot an pflegerischen Versorgungsstrukturen (Brettschneider 2019). In einigen Bundesländern können die Kommunen durch die Investitionsbedingungen einen begrenzten Einfluss z. B. auf stationäre Pflegeeinrichtungen nehmen, doch geht es dabei vorrangig um eine Eingrenzung des Angebots. Im Kontext der KZP wird hingegen ein Ausbau des Angebots angestrebt, da diese zur Entlastung pflegender Angehöriger und somit wiederum zur Stabilisierung häuslicher Pflegearrangements beitragen kann (Brettschneider 2019).

\section{- Ansätze zur quantitativen Ausweitung des Angebots an KZP-Plätzen}

2018 wurde in NRW die sogenannte „Fix/ Flex-Regelung“ für eingestreute KZP beschlossen. Mittels einer finanziellen Förderung sollen Anreize für Pflegeeinrichtungen geschaffen werden, einzelne eingestreute Kurzzeitpflegeplätze in solitäre (fixe) KZP-Plätze umzuwandeln (Tillmann und Sloane 2018). Neben solchen Konzepten, die darauf zielen, Anreize auf Seiten der Pflegeeinrichtungen zu schaffen, bestehen Bestrebungen, KZP in Krankenhäusern durch die Krankenhäuser selbst anzubieten (siehe - Tab. 9.2). Derzeit existiert z. B. in NRW ein Versorgungsvertrag zwischen den Landesverbänden der Pflegekassen und dem Landschaftsverband Rheinland zur „Erprobung von Kurzzeitpflege im Krankenhaus“ als Modellprojekt nach $§ 72$ SGB XI 
(ebd.). Auch wurden während der COVIDPandemie 2020 Reha-Kliniken für die KZP geöffnet (Deutscher Bundestag 2020).

Beide Konzepte zielen darauf, das Angebot an KZP-Plätzen quantitativ auszuweiten. Grundlage bilden im Wesentlichen wirtschaftliche Anreize für die Einrichtungen respektive Krankenhäuser. Aussagen darüber, ob die Anreize ausreichen, um das bestehende Angebot zu erweitern, sind bislang schwierig, da es aus unterschiedlichen Gründen an abschließenden Evaluationen mangelt. Des Weiteren bestehen strukturelle Hürden bei der Umsetzung der Konzepte, wie etwa die Konkurrenz zwischen KZP- und Dauerpflegeplätzen. Betriebswirtschaftlich ist es für Krankenhäuser nicht rentabel, wenn Patientinnen und Patienten Krankenhauskapazitäten blockieren, während auf freie KZP-Plätze gewartet wird, gleichzeitig sind Krankenhäuser seit 2015 durch das GKVVersorgungstrukturgesetz nach $§ 39$ Abs. 1a SGB V dazu verpflichtet ein Entlassmanagement durchzuführen, das auch die Gewährleistung der (pflegerischen) Anschlussversorgung beinhaltet. Entsprechend stellt sich die Nutzung von leerstehenden Krankenhausbetten als KZP-Plätze als sinnvoll dar. Gleichwohl bestehen auch hier strukturelle, organisatorische sowie förderrechtliche Hürden, die betriebswirtschaftliche Einschränkungen mit sich bringen, u.a. mit Blick auf Auslastungsquoten, Investitionskosten, die Verrechnung von Erlösen aus den Pflegekosten mit dem Pflegebudget des Krankenhauses sowie den Aspekt, dass die medizinische Versorgung in der $\mathrm{Zu}-$ ständigkeit der vertragsärztlichen Versorgungliegt. ${ }^{2}$

Versorgungsmanagement im Sinne der „Weichenstellerfunktion“ nach § 42 SGB XI respektive § 39c SGB V und weiterführend therapeutische, aktivierende Ansätze sind in beiden Ansätzen hingegen nicht im Spezifi-

2 Aufgrund der landesspezifischen Gesetzgebungen ist eine solche Nutzung nicht in jedem Bundesland möglich. Bislang war eine Nutzung auch nur dann möglich, wenn eigenständige Organisationen, die jedoch an ein Krankenhaus angegliedert sein konnten, gebildet wurden. schen vorgesehen und aufgrund der Einbettung in die bestehenden sozialrechtlichen Strukturen auch nur eingeschränkt möglich.

\section{- neue Versorgungsansätze}

Versorgungsansätze, die über die aktuell bestehenden Versorgungsstrukturen hinausgehen, spiegeln sich u.a. im "Pflege-Plus“"Konzept des AOK-Bundesverbandes oder in der vom Innovationsfonds des Gemeinsamen Bundesausschusses (G-BA) geförderten „REKUP“-Studie wider (Grund et al. 2020).

Grundlage des Pflege-Plus-Konzeptes bildet die Versorgung durch ein spezialisiertes ambulant-geriatrisches Versorgungsteam, das in interdisziplinärer Zusammenarbeit eine stärkere Verknüpfung des ambulanten, teilstationären und vollstationären Sektors ermöglichen und durch eine aktivierend-therapeutische Pflege die Autonomie und die Partizipation der Pflegebedürftigen in Abhängigkeit von ihren individuellen Ressourcen stärken soll (Ehmen et al. 2019). In der REKUP-Studie wird ein multidisziplinärer rehabilitativer Ansatz im Kurzzeitpflegeprozess - rehabilitative KZP - evaluiert. Geriatrische Patientinnen und Patienten erhalten direkt nach ihrem Krankenhausaufenthalt die rehabilitative KZP, mit dem Ziel, Rehabilitationsfähigkeit herzustellen sowie unerwünschte Ereignisse wie Komplikationen und Krankenhauseinweisungen oder die Überleitung in die vollstationäre Dauerpflege $\mathrm{zu}$ vermeiden. Beiden Ansätzen ist gemein, dass sie einer Reform der gesetzlichen Rahmenbedingungen bedürfen. Die finanziellen Aufwendungen z. B. für Physio- und Ergotherapie sowie Logopädie zur Unterstützung der aktivierend-therapeutischen Pflege werden bislang nicht durch die Pflegekassen getragen, hier steht u.a. die Trennung zwischen SPV und GKV einer Finanzierung im Wege. Auch fehlt es an Vergütungsmöglichkeiten für koordinierte niedrigschwellige Angebote zur Stärkung, Wiedererlangung und zum Erhalt der Selbstständigkeit der Patientinnen und Patienten für den stationären Bereich, damit diese nicht durch die Pflegebedürftigen selbst getragen werden müssen. Die existierenden 


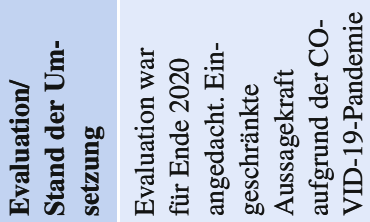

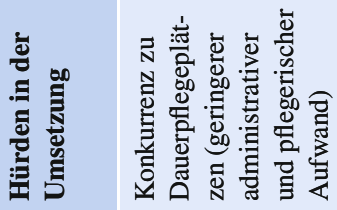

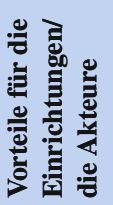

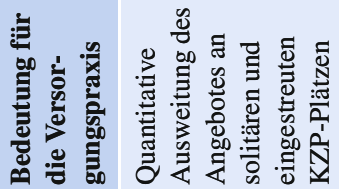

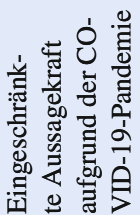
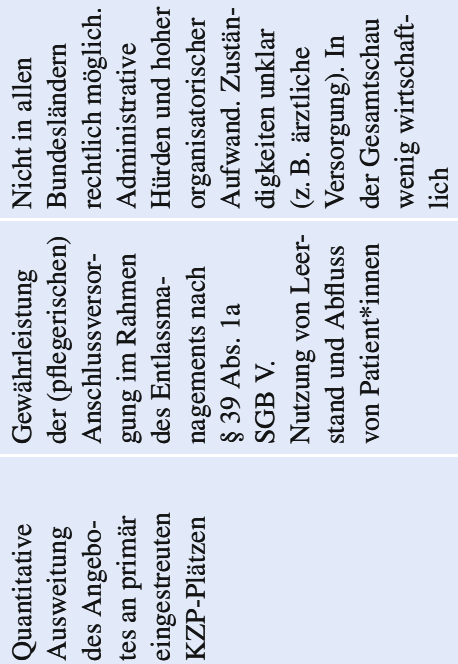

霜

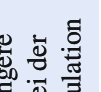

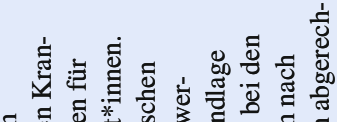

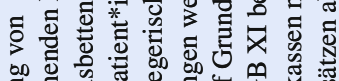
务

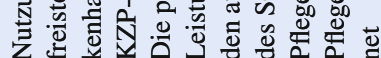
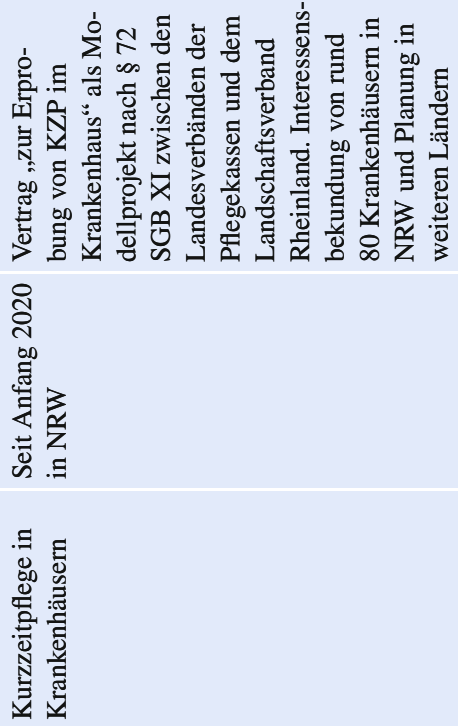


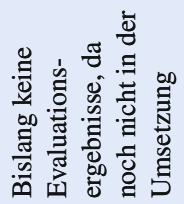

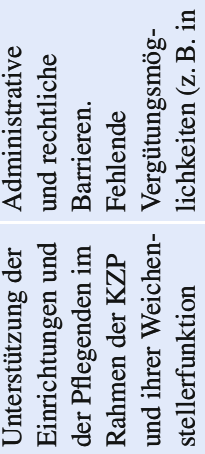

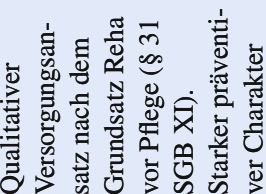

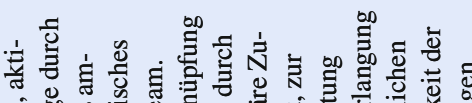

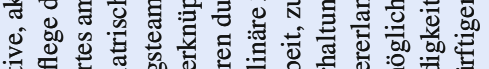

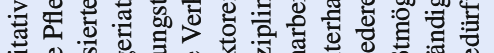

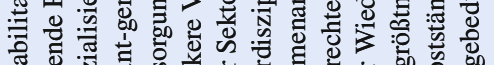

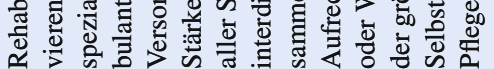

壱

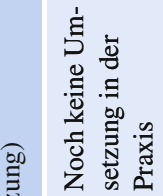

竞

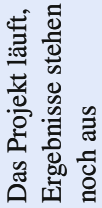

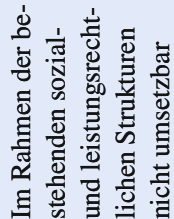

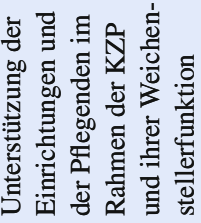

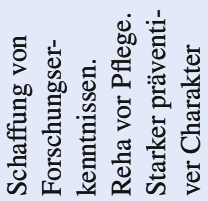

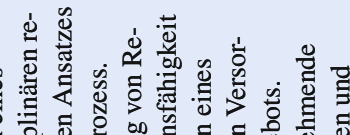

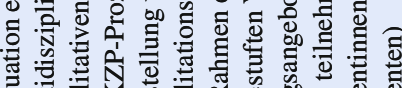

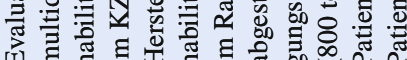

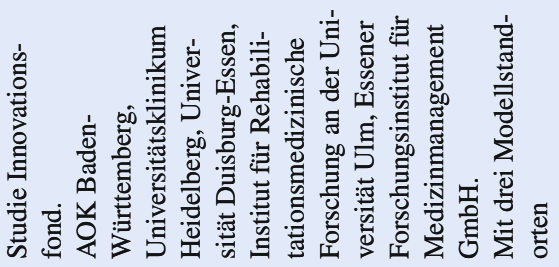

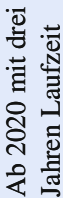

常 
(Pflege-)Pauschalen reichen für solche Versorgungskonzepte derzeit nicht aus, auch eine Umwidmung von finanziellen Mitteln aus dem Bereich der Prävention (u. a. $§ 5$ SGB XI) ist nach der aktuellen Gesetzeslage nicht möglich. Mit Inkrafttreten des Präventionsgesetzes (PrävG) hat die soziale Pflegeversicherung einen konkreten Präventionsauftrag erhalten, gesundheitsfördernde Angebote in stationären Pflegeeinrichtungen zu etablieren. Diese lassen sich jedoch vergütungstechnisch nicht mit kurativen, rehabilitativen oder anderen Versorgungsansätzen verknüpfen. Die bestehenden Ansätze zeigen ferner auf, dass der Bedarf an wissenschaftlich fundierten Kenntnissen über die Möglichkeiten von KZP als Versorgungsansatz mit unterschiedlichen Ausrichtungen und besonders mit dem Ziel der Herstellung von Rehabilitationsfähigkeit groß ist.

\subsection{Fazit}

Die KZP trägt in ihrer Funktion deutliche präventive und rehabilitative Ansätze und weist eine besondere Stellung innerhalb der Strukturen der pflegerischen Versorgung auf. Die oftmals ausgeprägte Heterogenität der KZP-Patientinnen und -Patienten geht mit einem erhöhten Bedarf an Personal und an multiprofessioneller Zusammenarbeit einher. Hinzu kommt die kurze Verweildauer und die damit verbundenen häufigen Wechsel an KZP-Patientinnen und -Patienten, die sowohl mit pflegerisch und therapeutisch anspruchsvollen Aufgaben als auch mit geringer wirtschaftlicher Attraktivität für das Betreiben von KZP-Einrichtungen oder -Plätzen einhergehen. Während die Unterschiede zwischen KZP und Langzeitpflege aufgrund ihres spezifischen Versorgungsauftrages in der Praxis deutlich erkennbar sind, ist die Unterscheidung in den leistungsrechtlichen Grundlagen kaum wiederzufinden.

Trotz Defiziten in der Statistik erscheint der Bedarf an KZP-Plätzen unzureichend gedeckt. Verbindliche Standards der örtlichen Pflegebedarfsplanung sind notwendig, um strukturelle Defizite in der pflegerischen Versorgung aufdecken und beheben zu können. Unter der Vorgabe, die Weichenstellerfunktion und damit das präventive Potenzial der KZP zu stärken, erscheinen Angebote an solitären KZP-Plätzen sinnvoller als eingestreute KZP-Plätze. Ob solitäre KZP-Einrichtungen auch einem aktivierenden respektive rehabilitativen Versorgungsansatz besser gerecht werden, erscheint zwar plausibel, bedarf aber weiterer Forschung. Demgegenüber steht die (Un)wirtschaftlichkeit solitärer KZP-Einrichtungen. Im Zuge der Sicherstellung der pflegerischen Versorgung wird vor allem der Ausbau solitärer, dauerhaft verfügbarer KZPPlätze diskutiert. Zentrale Voraussetzung dafür ist eine wirtschaftlich tragfähige Finanzierung der Einrichtung sowie qualifiziertes Fachpersonal (Deckenbach und Pflug 2019). Der unklare Versorgungsauftrag der KZP muss konkretisiert werden, mit Blick auf die Weichenstellerfunktion sowie mit klarer Abgrenzung zur Verhinderungs- und Dauerpflege. Dabei muss das gesetzlich verankerte Ziel, Pflegebedürftige in die Häuslichkeit zurückzuführen, im Sinne eines rehabilitativen Ansatzes stärker fokussiert werden.

Notwendig ist es daher, nicht nur den rein quantitativen Ausbau an KZP-Plätzen zu forcieren, sondern ebenso die qualitative und inhaltliche Entwicklung von KZP-Angeboten anzustreben. Es bedarf neuer Strukturen und Versorgungskonzepte, welche die spezifischen Bedarfe einzelner Gruppen von Patientinnen und Patienten und besonders den vielfach hohen Bedarf an Leistungen der medizinischen Rehabilitation im KZP-Kontext in den Blick nehmen.

Ob dies in naher Zukunft umgesetzt wird, bleibt abzuwarten, auch wenn bereits im Koalitionsvertrag 2018 eine Stärkung der KZP angekündigt und in einem aktuellen Papier des Bundesministeriums für Gesundheit (BMG) „Pflegeversicherung neu denken: Eckpunkte der Pflegereform 2021“ (BMG 2020; Stand 04.11.2020) aufgegriffen wurde. Die im Eckpunktepapier formulierten Forderungen gehen in die richtige Richtung, halten hingegen zu 
sehr an bestehende Strukturen fest und gehen in Teilen nicht weit genug. Eine Flexibilisierung der Leistungen der SPV zur Entlastung der betroffenen Haushalte (im Sinne eines Entlastungsbudgets) ist grundsätzlich zu unterstützen. Gleichzeitig braucht es aber einen neuen, erweiterten Versorgungsansatz im Sinne einer KZP, die auch präventiven und rehabilitativen Bedarfen gerecht wird, damit der Verbleib in der eigenen Häuslichkeit ermöglicht wird. Bisher fehlt es an einem klaren Versorgungsauftrag für die KZP, der auch einer klaren inhaltlichen Trennung zu anderen Versorgungsarten wie der Verhinderungspflege bedarf. Überdies sollte beachtet werden, dass die qualifizierte KZP in der Phase nach einem Krankenhausaufenthalt zwar hoch relevant ist, jedoch nicht auf diesen Versorgungsbereich reduziert werden sollte.

\section{Literatur}

Becker C, Auer R, Rapp K, Grund S, Bauer JM (2020) Geriatrische Rehabilitation - Aktueller Stand und zukünftige Entwicklung. In: Jacobs K, Kuhlmey A, Greß S, Klauber J, Schwinger A (Hrsg) Pflege-Report 2020: Neuausrichtung von Versorgung und Finanzierung. Springer, Berlin, S 135-148 https://doi.org/10.1007/ 978-3-662-61362-7

Blom S, Görres S (2012) Die ,neue“ Verantwortung der Kommunen - Herausforderungen für eine aktive politische Gestaltung zukunftsfähiger Versorgungsstrukturen für ältere Menschen. Informationsd Altersfr 39(2):3-10

BMG - Bundesministerium für Gesundheit (2020) Pflegeversicherung neu denken: Eckpunkte der Pflegereform 2021. 04.11.2020. https://www.barmer.de/ blob/271262/214a0bbbd4d9f04624b215a7559da9d9/ data/eckpunkte-pflegereform-2021.pdf (Erstellt: 1 . März 2021). Zugegriffen: 5. März 2020

BMG - Bundesministerium für Gesundheit (2021) Pflegeversicherung, Zahlen und Fakten. https://www. bundesgesundheitsministerium.de/themen/pflege/ pflegeversicherung-zahlen-und-fakten.html\#c3291. Zugegriffen: 12. März 2020

Braeseke G, Nauen K, Pflug C, Meyer-Rötz SH, Pisarek P (2017) Wissenschaftliche Studie zum Stand und zu den Bedarfen der Kurzzeitpflege in NRW. IGES Institut, Berlin (Abschlussbericht für das Ministerium für Arbeit, Gesundheit und Soziales des Landes Nordrhein-Westfalen)
Brettschneider A (2019) Die Rolle der Kommunen: Ziele, Handlungsfelder und Gestaltungsmöglichkeiten kommunaler Pflegepolitik. In: Jacobs K, Kuhlmey A, Greß S, Klauber J, Schwinger A (Hrsg) Pflege-Report 2019. Springer, Berlin Heidelberg, S 219-239 https://doi. org/10.1007/978-3-662-58935-9_18

CDU, CSU, SPD (2013) Deutschlands Zukunft gestalten: Koalitionsvertrag zwischen CDU, CSU und SPD, 18. Legislaturperiode. CDU, CSU, SPD, Berlin

Deckenbach B, Pflug C (2019) Modellerprobung „Überleitungsmanagement und Behandlungspflege in der Kurzzeitpflege“. IGES Institut, Berlin (Abschlussbericht für das Bundesministerium für Gesundheit)

Deckenbach B, Stöppler C, Klein S (2013) Qualitätskriterien für eine fachgerechte Kurzzeitpflege ( $\$ 42$ SGB XI). Studienbericht. Bundesministerium für Gesundheit, Berlin

Deutscher Bundestag (2019) Kurzzeitpflege stärken und eine wirtschaftlich tragfähige Vergütung sicherstellen. Drucksache 19/16045

Deutscher Bundestag (2020) Antwort der Bundesregierung auf die Kleine Anfrage der Abgeordneten Dr. Andrew Ullmann, Nicole Westig, Michael Theurer, weiterer Abgeordneter und der Fraktion der FDP Drucksache 19/22132. Drucksache 19/22847

Ehmen Y, Schwinger A, Tsiasioti C (2019) Neue Finanzierung der geriatrischen Reha. Mehr Rehabilitation für Pflegebedürftige? In: Szepan N-M, Wagne F (Hrsg) Agenda Pflege 2021, Grundlagen für den fachpolitischen Diskurs, S 175-194

Grund S, Keilhauer A, Trumpfheller A, Specht-Leible N, Bauer J (2020) REKUP Rehabilitative Kurzzeitpflege im stationären Umfeld. https://www. dvfr.de/fileadmin/user_upload/DVfR/Downloads/ Veranstaltungen/200117_MoRe/2020_0117_Grund_ REKUP_PPT_bf.pdf. Zugegriffen: 1. März 2021

Hackmann T, Müller D, Steiner M, Tiessen J (2014) Pflege vor Ort gestalten und verantworten. Konzept für ein Regionales Pflegebudget. Bertelsmann Stiftung, Gütersloh

Hartmann E (2002) Teil I: Kurzzeitpflege und Regionalstruktur. In: Hartmann E, Heinemann-Knoch M (Hrsg) Kurzzeitpflege in der Region. Bundesministerium für Familie, Senioren, Frauen und Jugend. Kohlhammer, Stuttgart

Heinzen J, Dreckmann J, Lambertz L, Klemm B (2020) Kurzzeitpflege in Krankenhäusern - ein Erfolgsmodell? Krankenhaus 5:404-406

Janßen H (2018) Ermittlung des allgemeinen Rehabilitationsbedarfs und Evaluation Mobiler Geriatrischer Rehabilitation in stationären Pflegeeinrichtungen und der Kurzzeitpflege Abschlussbericht, 10 September2018

Naegele G (2010) Kommunen im demografischen Wandel. Thesen zu neuen An- und Herausforderungen für die lokale Alten- und Seniorenpolitik. Z Gerontol Geriat 43:98-102 
Rothgang H, Müller R, Unger R (2012) Themenreport „Pflege 2030“. Was ist zu erwarten - was ist zu tun? Bertelsmann, Bielefeld

Rothgang H, Müller R, Unger R (2014) BARMER GEK Pflegereport 2014. Schwerpunkt: Zahnärztliche Versorgung Pflegebedürftiger. Schriftenreihe zur Gesundheitsanalyse, Bd. 29. BARMER GEK

Rothgang H, Kalwitzki T, Müller R, Runte R, Unger R (2015) Barmer GEK Pflegereport 2015. Schwerpunktthema: Pflege zu Hause. Schriftenreihe zur Gesundheitsanalyse, Bd. 36. BARMER GEK

Statistisches Bundesamt (2021) Pflegestatistik - Pflegeheime (Anzahl). Gliederungsmerkmale: Jahre, Deutschland, Pflegeangebot, Träger, Kapazitätsgrößenklassen Gesundheitsberichterstattung des
Bundes. https://www.gbe-bund.de/gbe/pkg_isgbe5. prc_menu_olap?p_uid=gast $\% 26 ;$ p_aid $=94255203$ $\% 26 ;$ p_sprache=D\%26;p_help=0\%26;p_indnr $=397$ $\% 26 ;$;_indsp=\%26;p_ityp=H\%26;p_fid. Zugegriffen: 1. März 2021

Sachverständigenrat zur Begutachtung der Entwicklung im Gesundheitswesen (2014) Bedarfsgerechte Versorgung - Perspektiven für ländliche Regionen und ausgewählte Versorgungsbereiche. SVR, Bonn, Berlin (Gutachten 2014)

Tillmann R, Sloane K (2018) Wann sich die Umsetzung für Heime lohnt. CAREkonkret 21:6

Vonau M Engpässe: Kurzzeitpflege neu denken. https:// www.aok.de/pk/nordwest/inhalt/kurzzeitpflege-neudenken/. Zugegriffen: 1. März 2021

Open Access Dieses Kapitel wird unter der Creative Commons Namensnennung 4.0 International Lizenz (http:// creativecommons.org/licenses/by/4.0/deed.de) veröffentlicht, welche die Nutzung, Vervielfältigung, Bearbeitung, Verbreitung und Wiedergabe in jeglichem Medium und Format erlaubt, sofern Sie den/die ursprünglichen Autor(en) und die Quelle ordnungsgemäß nennen, einen Link zur Creative Commons Lizenz beifügen und angeben, ob Änderungen vorgenommen wurden.

Die in diesem Kapitel enthaltenen Bilder und sonstiges Drittmaterial unterliegen ebenfalls der genannten Creative Commons Lizenz, sofern sich aus der Abbildungslegende nichts anderes ergibt. Sofern das betreffende Material nicht unter der genannten Creative Commons Lizenz steht und die betreffende Handlung nicht nach gesetzlichen Vorschriften erlaubt ist, ist für die oben aufgeführten Weiterverwendungen des Materials die Einwilligung des jeweiligen Rechteinhabers einzuholen.

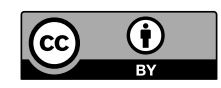

